\title{
Minimally invasive endoscopic approach to perform complete coccygectomy in patients with chronic refractory coccydynia: illustrative case
}

\author{
Jorge A. Roa, MD, ${ }^{1}$ Sarah White, BS, ${ }^{2}$ Ernest J. Barthélemy, MD, MA, MPH, ${ }^{3}$ Arthur Jenkins III, MD, ${ }^{1}$ and \\ Konstantinos Margetis, MD, $\mathrm{PhD}^{1}$ \\ ${ }^{1}$ Department of Neurosurgery, Icahn School of Medicine at Mount Sinai, New York, New York; ${ }^{2}$ Campbell University School of Osteopathic Medicine, Lillington, North Carolina; \\ and ${ }^{3}$ Department of Neurosurgery, University of California, San Francisco, San Francisco, California
}

BACKGROUND Coccydynia refers to debilitating pain in the coccygeal region of the spine. Treatment strategies range from conservative measures (e.g., ergonomic adaptations, physical therapy, nerve block injections) to partial or complete removal of the coccyx (coccygectomy). Because the surgical intervention is situated in a high-pressure location close to the anus, a possible complication is the formation of sacral pressure ulcers and infection at the incision site.

OBSERVATIONS In this case report, the authors presented a minimally invasive, fully endoscopic approach to safely perform complete coccygectomy for treatment of refractory posttraumatic coccydynia.

LESSONS Although this is a single case report, the authors hope that this novel endoscopic approach may achieve improved wound healing, reduced infection rates, and lower risk of penetration injury to retroperitoneal organs in patients requiring coccygectomy.

https://thejns.org/doi/abs/10.3171/CASE21533

KEYWORDS coccydynia; refractory; coccygectomy; endoscopic; minimally invasive; surgical approach; novel technique

Coccydynia refers to debilitating pain in the coccyx region of the spine. The term was first adopted by Simpson in 1859, and it is derived from two Greek words: coccyx (resemblance to a cuckoo's beak) and dynia (pain). ${ }^{1}$ At least six different etiologies have been identified as potential causes/risk factors to develop coccydynia:

1. Trauma: History of direct coccygeal trauma (typically after a backward fall into a seated position) has been found to be present in 50\%-65\% of patients with coccydynia. Depending on the severity of the trauma, the injury may include a sprain of the pelvic floor muscles, mild distortion over a fissure in the caudal coccygeal segment, or a severe fracture-dislocation of the sacrococcygeal complex.

2. Obesity and female gender: The biomechanical basis that correlates coccydynia with obesity has been attributed to restricted sagittal pelvic rotation in obese individuals while sitting, leading to protrusion, retroversion, and excessive pressure over the distal tip of the coccyx. ${ }^{2}$ Additionally, increased intrapelvic pressure during prolonged sitting may lead to posterior subluxation of the jutting out tip of the coccyx. Women are five times more prone than men to develop coccydynia because of inherent ligamentous laxity, susceptible coccygeal morphology, and childbirth. ${ }^{3}$

3. Coccygeal morphology: Based on its curvature, the anatomy of the sacrococcygeal junction was categorized into four different configurations by Postacchini and Massobrio in 1983, ${ }^{4}$ with two more types further added by Nathan et al. ${ }^{3}$ : type I, minor anterior curvature; type II, noticeable anterior curvature with the apex pointing straight forward; type III, sharp angulation at the superior intercoccygeal joints; type IV, subluxation between segments; type $V$, retroverted appearance with posteriorly angulated apex; and type VI, scoliotic or laterally subluxated coccyx. The prevalence of these anatomical configurations varies among different populations. Types III to $\mathrm{VI}$ are less prevalent but have a significantly higher incidence of coccydynia, especially when associated with the presence of a bony spicule at the coccygeal tip.

ABBREVIATIONS CT = computed tomography; MRI = magnetic resonance imaging; VAS = visual analog scale. INCLUDE WHEN CITING Published January 17, 2022; DOI: 10.3171/CASE21533.

SUBMITTED September 15, 2021. ACCEPTED November 29, 2021.

(C) 2022 The authors, CC BY-NC-ND 4.0 (http://creativecommons.org/licenses/by-nc-nd/4.0/). 
4. Coccygeal hypermobility: Abnormal coccygeal translation (i.e., angular motion in dynamic standing and seated radiographs) has been found in $69 \%$ of patients presenting with coccydynia. Maigne et al. ${ }^{2}$ classified coccygeal mobility into four types: (1) luxation: posterior subluxation of the mobile segment of coccyx on assuming sitting posture; (2) hypermobility: direct seating axial pressure leading to coccygeal flexion $>25^{\circ}$; (3) immobile: $<5^{\circ}$ of coccygeal flexion on sitting; and (4) normal: coccygeal mobility of $5^{\circ}$ to $25^{\circ}$.

5. Discogenic/articular pain: Although fused in most cases, intercoccygeal articulations contain intervertebral disc. Degenerative changes have been implicated as a potential cause of coccydynia in $41 \%$ of idiopathic cases. In a histological study by Balain et al., five of eight patients who received coccygectomy demonstrated degenerative disc changes whereas two had degenerative articular cartilage changes.

6. Miscellaneous: Previous literature has reported other rare causes of coccydynia, such as tuberculosis, tumors such as chordoma, osteoid osteoma, trophoblastic carcinoma, notochordal cell tumors, excessive calcium salts deposition in intercoccygeal or sacrococcygeal articulations, and neuropsychosis.,

Diagnostic imaging in coccydynia cases can be easily interpreted once the pathophysiology has been discussed. Lateral and anteroposterior radiographs constitute the most important first step to rule out infections, tumors, or predisposed coccygeal morphology, such as retroverted coccyx, bony spicule, coccygeal scoliosis, or subluxation. In cases of trauma, in which radiographs are suggestive of a fracture or dislocation, computed tomography (CT) is recommended for definitive diagnosis. In patients with normal static imaging, dynamic imaging may be performed to rule out abnormal coccygeal mobility. As discussed, an angular mobility $>25^{\circ}$ or $<5^{\circ}$ is considered abnormal. At least two nonstandardized methods have been described to measure angular mobility: the angle formed by two lines drawn from the center of the last mobile segment to the tip of the coccyx in superimposed films (according to Maigne et al. ${ }^{2}$ ) and the angle between two lines drawn parallel and bisecting the first and last segment of coccyx (according to Kim et al. ${ }^{8}$ ).

Magnetic resonance imaging (MRI) is useful to identify areas of signal alteration in and around the coccyx that may explain the origin of the pain. Areas of T2 hyperintensity with low T1 signals (Modic type 1 changes) suggest fissuring of the endplates and development of adjacent vascular granulation tissue with bone marrow edema. ${ }^{9}$ MRI may also be able to diagnose chronic irritation and bursitis on the tip of coccyx or the soft tissue posteriorly.
Treatment strategies for coccydynia aim to manage its primary cause. In general, these modalities may be classified as follows: ergonomic adaptation (doughnut or ring-shaped pillows, posture training, buttock strapping, stool-softening measures), physical therapy (manipulation of coccyx and massage of the levator ani [pelvic floor muscles]); nerve block injections (steroid or anesthetic injections, prolotherapy, ganglion impar block), and surgery (coccygeoplasty or partial/complete coccygectomy).

In this case report, we discuss a minimally invasive endoscopic approach to safely perform complete coccygectomy for treatment of refractory posttraumatic coccydynia.

\section{Illustrative Case \\ History and Examination}

A male patient in his 60 s without significant past medical history presented with 1 year of progressively worsening right-sided pain to the lower back and gluteal region after a fall. The pain was sharp and nonradiating, with an intensity of $10 / 10$ on the visual analog scale (VAS), worsened with sitting, and improved when lying down. The patient's pain modestly and transiently improved with acetaminophen; however, it quickly returned after 1 or 2 hours of relief. He denied any bowel/bladder incontinence or saddle anesthesia.

Further conservative management was pursued via ganglion impar blocking performed by a pain management specialist. ${ }^{10}$ The injection initially resulted in complete resolution of the pain; however, the pain returned after a mechanical fall 2 months later while attempting to reach for an object high on a shelf, complicated by direct impact to his tail bone. Physical examination was notable for significant tenderness to palpation of the sacrococcygeal region. His neurological examination was otherwise unremarkable, and laboratory study results were within normal limits.

Standing radiographs and CT films of the sacrum demonstrated a posterior step-off of the coccyx (Fig. 1A and B) with sclerotic margins; increased posterior subluxation of the coccyx was noticed on sitting films when compared with standing views. An MRI of the pelvis without contrast showed arthropathy of the coccyx with mild separation between the first and second coccygeal segments and dorsal subluxation of the distal segment by $5 \mathrm{~mm}$ (Fig. 1C). Periarticular marrow edema associated with mild surrounding bony productive change was also noted. Additionally, there were signs of trace joint effusion and presacral soft tissue edema. After informed consent was obtained, the
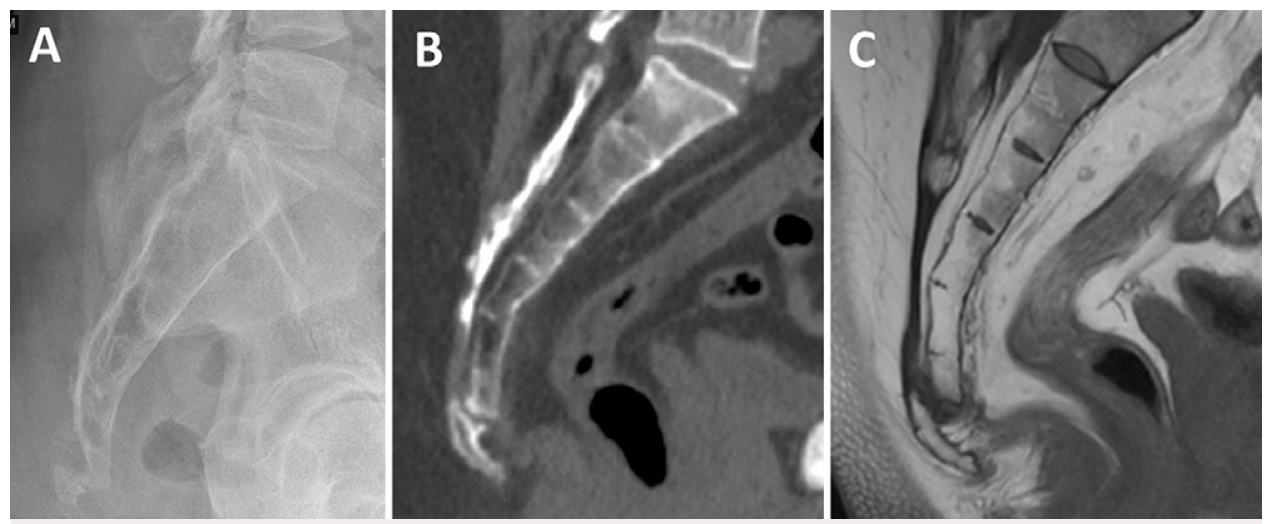

FIG. 1. Lateral/sagittal films of a patient with posttraumatic coccydynia demonstrating dorsal subluxation of the tip of the coccyx: radiographs $(\mathbf{A}), \mathrm{CT}(\mathbf{B})$, and MRI (C). 


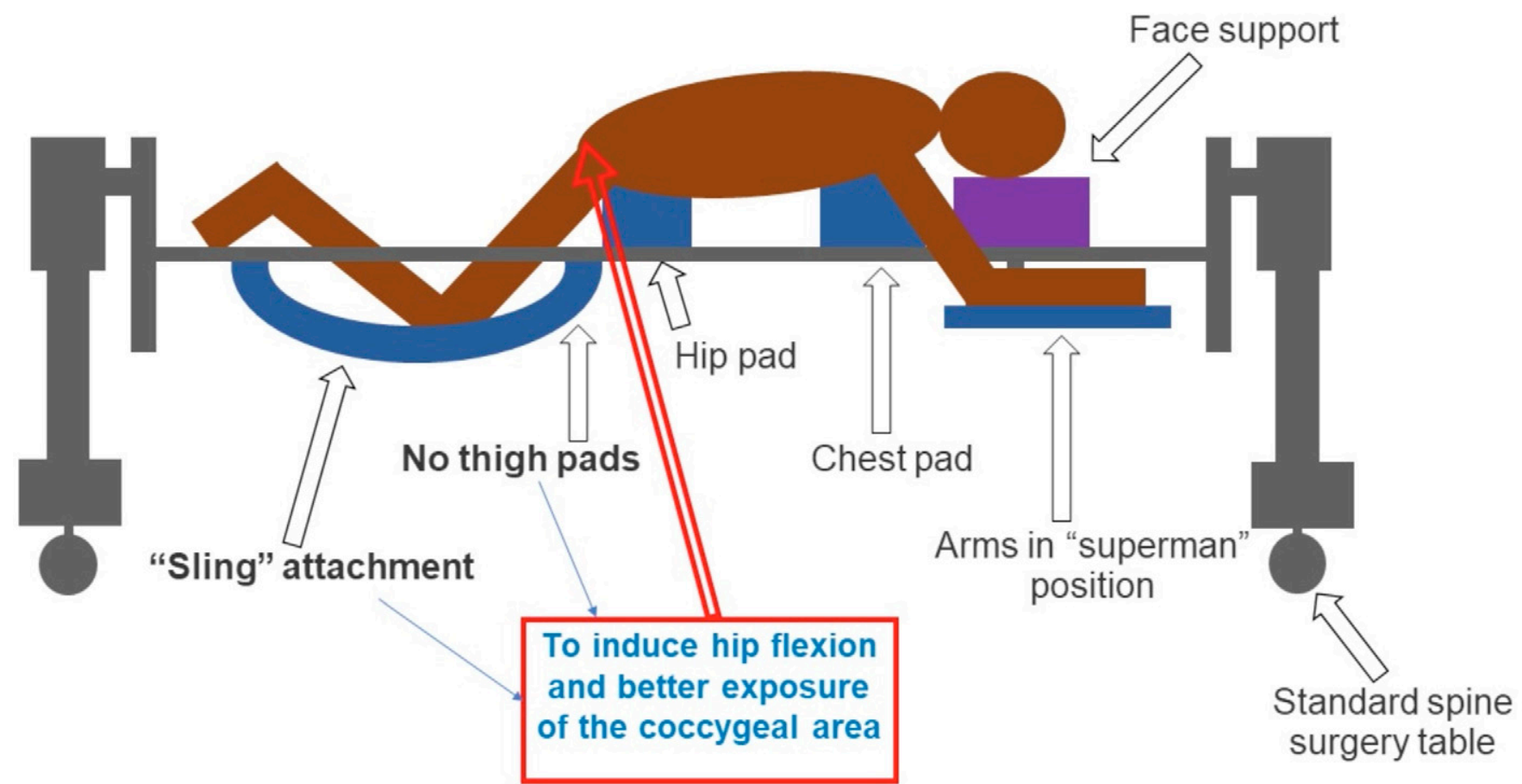

FIG. 2. Scheme depicting the prone position of the patient to perform endoscopic coccygectomy. It is worthwhile noticing that we do not place the patient's thighs on a pad but rather use a "sling attachment" to induce additional hip flexion and maximize the surgical exposure of the coccygeal area.

patient successfully received complete coccygectomy using our proposed minimally invasive endoscopic approach.

\section{Endoscopic Coccygectomy: Surgical Technique}

A video describing the technique can be found as part of the Supplementary Material (Video 1). A step-by-step description is presented in the following paragraphs.

\section{Positioning and Localization}

After intubation, the patient was placed prone onto the Jackson table with the lower extremities positioned in the leg sling so that the hips and knees were both bent (Fig. 2). Lateral fluoroscopic images were obtained intraoperatively for localization purposes (Fig. 3A).

\section{Incision and Insertion of Endoscope}

Local anesthesia was infiltrated into the subcutaneous and subperiosteal regions. A\#15 blade was then used to perform a stab incision, and a monopolar electrocautery was used to dissect subcutaneous tissue, palpate the coccyx, and perform a subperiosteal dissection on the dorsal surface of the coccyx. Of note, the electrocautery was set to low power to prevent conduction through the tissues into the retroperitoneal contents. Then, the endoscopic system dilator was inserted, and a second lateral radiograph was obtained to confirm accurate placement of the endoscopic dilator (Fig. 3B). The dilator was removed and the endoscope was advanced through the endoscopic cannula.

\section{Endoscopic Drilling}

Under endoscopic visualization, we cleared the soft tissue over the coccyx at the sacrococcygeal junction using endoscopic pituitary rongeurs. Then, using a diamond drill bit, we performed resection of the coccyx as well as the caudal aspect of S5. Blunt instruments may be used to identify bone remnants along the edges of the coccyx using a combination of visualization and palpation (Video 1).

VIDEO 1. Clip showing our minimally invasive endoscopic approach to perform complete coccygectomy. Click here to view.

\section{Hemostasis and Closure}

After obtaining a lateral radiograph to confirm that the whole coccyx had been resected (Fig. 3C), hemostasis in the surgical bed was performed. The endoscope was removed, a Vicryl 3-0 suture was used to close the dermis, and a Monocryl 3-0 suture was run in a standard subcuticular fashion.

\section{Discussion}

The surgical procedure for coccygectomy has not changed in almost 90 years. Coccygectomy was initially described in 1937 by Key et al. ${ }^{11}$ as a last resort after conservative treatments failed to provide pain relief. The first documented technique involved a midline vertical incision over the sacrococcygeal joint approximately 2.5 inches long. Because the caudal end is usually pointed anteriorly, the sacrococcygeal joint is cut first and pulled posteriorly to expose the tip and remove the coccyx in one piece. ${ }^{11}$ Gardner ${ }^{12}$ attempted to improve this technique by removing the tip first and separating it from the rectum to prevent rectal damage and infection. ${ }^{7}$ However, previous reports have demonstrated that the rostral-to-caudal direction of the resection proposed by Key et al. achieves better outcomes. ${ }^{4}$ A transverse incision approach has also been used, but its scar formation produces aesthetically less favorable results for patients. ${ }^{8}$ Recently, a "Z" plasty technique was used in wound 

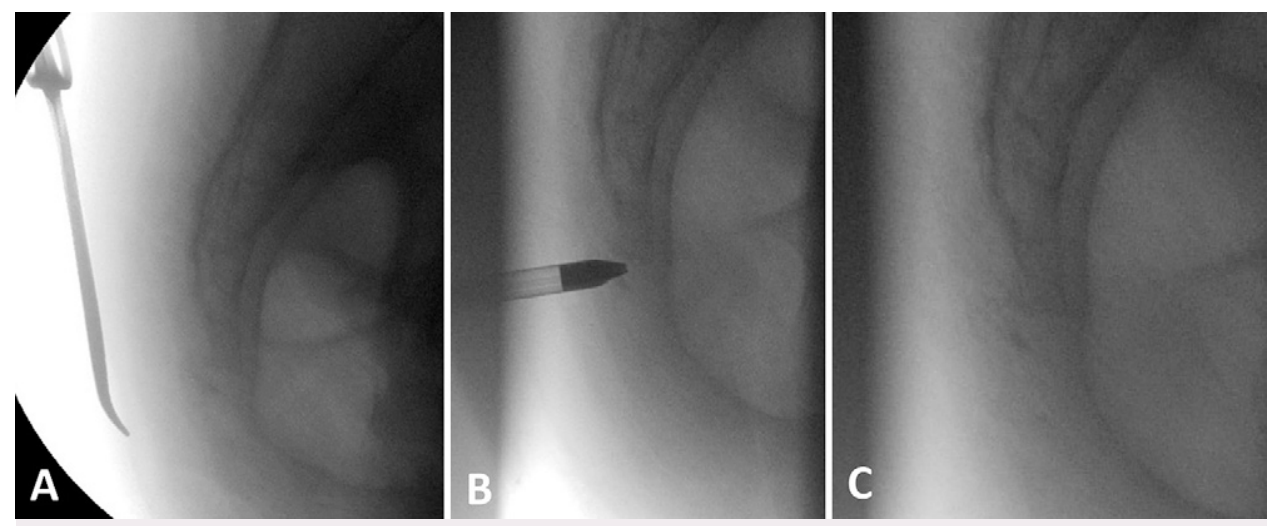

FIG. 3. Sequence of intraoperative fluoroscopic radiographs used to localize the coccygeal bone (A), confirm accurate placement of the endoscopic dilator in relationship to the sacrococcygeal junction (B), and confirm complete coccygectomy $(\mathbf{C})$.

closure to the distal end of the vertical incision to reduce wound dehiscence and infection rates. ${ }^{13}$ In this technique, considering the primary vertical incision as the central limb of the "Z," the incision is extended in opposite directions at an angle of $60^{\circ}$. Then, the flaps are raised such that the surgical wound looks like a parallelogram. Finally, meticulous apposition of the flaps without tension is achieved using interrupted subcutaneous 2-0 Vicryl stitches. ${ }^{13}$

Many studies have confirmed the effectiveness of coccygectomy in accomplishing pain control in most cases. ${ }^{14-20}$ However, pain-free rates postcoccygectomy may be influenced by the specific etiology causing coccydynia. In a study of 48 patients, Bayne et al. ${ }^{21}$ showed that coccygectomy is rarely successful for pain associated with lumbar disc disease requiring lumbar laminectomy and spinal fusion and reaches approximately $60 \%$ success in spontaneous idiopathic cases. Small modifications have been tested for the sake of better outcomes, fewer operative complications, and lower chance for infection. The endoscopic approach used in this case has the potential to reduce the size of the incision profoundly (from approximately 2 inches to approximately $8 \mathrm{~mm}$ ), which has implications for less infection risk and better wound healing. Because the surgical intervention is situated in a high-pressure location closed to the anus, the most common complications from the operation are sacral ulcers and infection at the incision site. ${ }^{4-6}$ Based on the coccyx types originally defined by Postacchini and Massobrio, our patient presented with a type IV, which involves subluxation between segments.

There is no definite evidence to suggest that complete resection leads to better outcomes and pain control compared with partial coccygectomy. In a retrospective study by Ogur et al., ${ }^{22} 14$ patients received partial coccygectomy, whereas eight received total resection via open technique. No statistically significant difference was determined between the two groups with respect to the mean pain scores using VAS ( $p>0.05)$. Excellent and good pain control was obtained in $78 \%$ of all patients, with an overall satisfaction rate of $90 \%$.

\section{Observations}

The two most significant complications are related to wound complications (e.g., difficult wound healing/increased risk of infection) and injury to the structures ventral to the coccyx, including the rectum, blood vessels, bladder, and reproductive organs. The location of the procedure in a high-pressure region often results in decreased perfusion of the healing tissue as well as high risk of colonization from proximity to the anus. The endoscopic approach has the potential to reduce the size of the incision significantly (from $\geq 2$ inches to approximately $8 \mathrm{~mm}$ ), which tends to correlate with reduced infection risk. The endoscopic technique would allow for the surgeon to select an incision away from the usual pressure points and specifically more cranially but still have the instruments be within reach of the target area. In addition, performing the procedure under the high magnification of a modern endoscopic camera system reduces the risk of iatrogenic penetration injury past the coccyx into the retroperitoneal space with the surgical instruments. The case presented in this report, although performed under general anesthesia, could also have been completed under local anesthesia with sedation. With the coccyx removed, the patient was able to resume his normal activities after a period of 3 weeks.

\section{Lessons}

Endoscopic coccygectomy is a feasible and effective surgical approach for the treatment of coccydynia. Although this is a single case report, we hope that this surgical technique may achieve improved wound healing, reduced infection rates, and lower risk of penetration injury to retroperitoneal organs.

\section{References}

1. Simpson J. Coccygodynia and diseases and deformities of the coccyx. Med Times Gaz. 1859;40:1-7.

2. Maigne JY, Doursounian L, Chatellier G. Causes and mechanisms of common coccydynia: role of body mass index and coccygeal trauma. Spine (Phila Pa 1976). 2000;25(23):3072-3079.

3. Nathan ST, Fisher BE, Roberts CS. Coccydynia: a review of pathoanatomy, aetiology, treatment and outcome. J Bone Joint Surg Br. 2010;92(12):1622-1627.

4. Postacchini F, Massobrio M. Idiopathic coccygodynia. Analysis of fifty-one operative cases and a radiographic study of the normal coccyx. J Bone Joint Surg Am. 1983;65(8):1116-1124.

5. Balain B, Eisenstein SM, Alo GO, et al. Coccygectomy for coccydynia: case series and review of literature. Spine (Phila Pa 1976). 2006;31(13):E414-E420.

6. Mechri M, Riahi H, Sboui I, Bouaziz M, Vanhoenacker F, Ladeb M. Imaging of malignant primitive tumors of the spine. J Belg Soc Radiol. 2018;102(1):56. 
7. Blocker O, Hill S, Woodacre T. Persistent coccydynia: the importance of a differential diagnosis. BMJ Case Rep. 2011;2011: bcr0620114408.

8. Kim NH, Suk KS. Clinical and radiological differences between traumatic and idiopathic coccygodynia. Yonsei Med J. 1999;40(3): 215-220.

9. Maigne JY, Pigeau I, Roger B. Magnetic resonance imaging findings in the painful adult coccyx. Eur Spine J. 2012;21(10):2097-2104.

10. Gonnade N, Mehta N, Khera PS, Kumar D, Rajagopal R, Sharma PK. Ganglion impar block in patients with chronic coccydynia. Indian J Radiol Imaging. 2017;27(3):324-328.

11. Key JA. Operative treatment of coccygodynia. JBJS. 1937;19(3): 759-764.

12. Gardner RC. An improved technic of coccygectomy. Clin Orthop Relat Res. 1972;85(85):143-145.

13. Kulkarni AG, Tapashetti S, Tambwekar VS. Outcomes of coccygectomy using the "Z" plasty technique of wound closure. Global Spine J. 2019;9(8):802-806.

14. Ersen O, Ekinci S, Koca K, Akyildiz F, Bilgic S. Coccygectomy as a surgical option in the treatment of chronic traumatic coccygodynia. Asian Spine J. 2015;9(3):492.

15. Kalstad AM, Knobloch RG, Finsen V. Resection of the coccyx as an outpatient procedure. Orthop Rev (Pavia). 2020;12(3):8813.

16. Antoniadis $\mathrm{A}$, Ulrich $\mathrm{NH}$, Senyurt $\mathrm{H}$. Coccygectomy as a surgical option in the treatment of chronic traumatic coccygodynia: a singlecenter experience and literature review. Asian Spine J. 2014;8(6): 705-710.

17. Trollegaard AM, Aarby NS, Hellberg S. Coccygectomy: an effective treatment option for chronic coccydynia: retrospective results in 41 consecutive patients. J Bone Joint Surg Br. 2010;92(2):242-245.

18. Traub S, Glaser J, Manino B. Coccygectomy for the treatment of therapy-resistant coccygodynia. J Surg Orthop Adv. 2009;18(3): 147-149.

19. Sehirlioglu A, Ozturk C, Oguz E, Emre T, Bek D, Altinmakas M. Coccygectomy in the surgical treatment of traumatic coccygodynia. Injury. 2007;38(2):182-187.
20. Perkins R, Schofferman J, Reynolds J. Coccygectomy for severe refractory sacrococcygeal joint pain. J Spinal Disord Tech. 2003;16(1):100-103.

21. Bayne O, Bateman JE, Cameron HU. The influence of etiology on the results of coccygectomy. Clin Orthop Relat Res. 1984;(190): 266-272.

22. Ogur HU, Seyfettinoğlu F, Tuhanioğlu Ü, Cicek H, Zohre S. An evaluation of two different methods of coccygectomy in patients with traumatic coccydynia. J Pain Res. 2017;10:881-886.

\section{Disclosures}

The authors report no conflict of interest concerning the materials or methods used in this study or the findings specified in this paper.

\section{Author Contributions}

Conception and design: all authors. Acquisition of data: Margetis, Roa, Barthélemy, Jenkins. Analysis and interpretation of data: Margetis, Roa, Jenkins. Drafting the article: Roa, White, Barthélemy, Jenkins. Critically revising the article: Margetis, Roa, Barthélemy, Jenkins. Reviewed submitted version of manuscript: Margetis, Roa, Jenkins. Approved the final version of the manuscript on behalf of all authors: Margetis. Statistical analysis: Jenkins. Administrative/technical/material support: Barthélemy, Jenkins. Study supervision: Margetis, Jenkins.

\section{Supplemental Information \\ Video}

Video 1. https://vimeo.com/651596864.

\section{Correspondence}

Konstantinos Margetis: Icahn School of Medicine at Mount Sinai, New York, NY. konstantinos.margetis@mountsinai.org. 